\title{
Buonomano against Bell: Nonergodicity or nonlocality?
}

\author{
Andrei Khrennikov \\ International Center for Mathematical Modeling \\ Linnaeus University, Växjö, S-35195, Sweden \\ National Research University of Information Technologies, \\ Mechanics and Optics (ITMO), St. Petersburg, Russia
}

July 22,2018

\begin{abstract}
The aim of this note is to attract attention of the quantum foundational community to the fact that in Bell's arguments one can not distinguish two hypotheses: a) quantum mechanics is nonlocal, b) quantum mechanics is nonergodic. Therefore experimental violations of Bell's inequality can be as well interpreted as supporting the hypothesis that stochastic processes induced by quantum measurements are nonergodic. The latter hypothesis was discussed actively by Buonomano since 1980th. However, in contrast to Bell's hypothesis on nonlocality it did not attract so much attention. The only experiment testing the hypothesis on nonergodicity was performed in neutron interferometry (by Summhammer, in 1989). This experiment can be considered as rejecting this hypothesis. However, it cannot be considered as a decisive experiment. New experiments are badly needed. We point out that a nonergodic model can be realistic, i.e., the distribution of hidden (local!) variables is well defined. We also discuss coupling of violation of the Bell inequality with violation of the condition of weak mixing for ergodic dynamical systems.

keywords: Hypothesis of ergodicity, Bell inequality, time and ensemble averages, locality, realism, ontic and epistemic descriptions, ergodic theorems, weak mixing
\end{abstract}




\section{Introduction}

In 2015 a few respectable experimental groups claimed that they were able to close simultaneously the two basic loopholes in the Bell-test experiments [1] (see also 2] for review and references herein) the locality loophole and the fair sampling loophole, see [3]-5] These successful experiments may lead to the conclusion that the debate between Einstein [10] and Bohr [11] can be considered as finished and that the position of Einstein, Podolsky, and Rosen is not supported by the experimental evidence, see, e.g., [12]-[13]. However, not everybody agreed with such finalizing views [14, 15]. Personally I think that the main foundational problems related to Bell's argument have not yet been resolved and that further intensive theoretical and experimental studies are needed [15].

In this note we would like to discuss coupling of the Bell argument with the hypothesis on ergodicity of quantum mechanics. The main claim is that experimental violations of the Bell inequality need not necessarily suggest to reject local-realism, but rather ergodicity.

In physics nonergodicity (in particular, violation of the law of large numbers) is considered as a kind of pathology. Appearance of statistical data which deviate from ergodic behavior is considered as a sign that this experiment was not been well performed. However, this heuristics came from probabilistic analysis of macroscopic phenomena. In principle, there is no guarantee that it should work as well for quantum phenomena. The hypothesis about violation of ergodicity for such phenomena was actively discussed by Buonomano [16] since 1980th. Honestly saying, his works did not attract so much attention. The only experiment testing the hypothesis on nonergodicity was performed in neutron interferometry by Summhammer [17]. This experiment can be considered as rejecting this hypothesis and confirming ergodicity of quantum statistical data 2 However, it definitely cannot be considered as a decisive experiment rejecting completely the hypothesis on nonergodicity of quantum mechanics. New experiments

\footnotetext{
${ }^{1}$ Of course, the debate on loopholes can be continued for ever, see, e.g., the papers on signaling loophole 6 - 9 .

${ }^{2}$ And this experiment was practically forgotten. I got to know about it only from private conversations with J. Summhammer during his visits to Växjö - as one of curious things which he did when he was young. For example, by giving at least 10 talks about neutron interferometry at the Växjö conferences H. Rauch had never mentioned this experiment which was performed at his institute.
} 
are badly needed. In [18, 19] I proposed a few experimental tests for Buonomano's hypothesis 3 They were even discussed at Atominstitute in Vienna, but they have never been performed. Typically other experiments were considered as having higher priority.

In preprint 25] there was pointed out that Buonomano's hypothesis is related to Bell's argument. In fact, if Buonomano were right, then it would be impossible to derive the Bell inequality. From this viewpoint, observed experimentally violations of this inequality need not be interpreted as the crucial argument to reject local realism. These violations can be treated as a sign that quantum mechanics is nonergodic. The aim of the present paper is to attract attention of the quantum community to the nonergodic hypothesis and to the possibility to interpret the recent exciting loophole free Bell-experiments as simply supporting this hypothesis. We remark that in fact this hypothesis (but without explicit operating with the notion of (non)ergodicity) was discussed in connection to the Bell inequality in few papers, e.g., [21], [22].

Besides the philosophic and mathematical messages, this paper delivers the important message for experimenters: to perform a series of new experiments to test nonegodicity in quantum physics - experiments for the straightforward tests of nonegodicity, a la Summhammer.

In sections 6, 7 (which are more mathematical than the rest of the paper) we discuss coupling of the Bell argument with theories of ergodic stochastic processes [40] and dynamical systems [24]. This theories imply that dynamics behind joint measurements on components of a compound quantum system $S$ need not be ergodic, in spite of ergodicity of the dynamics behind measurements on the subsystems $S_{j}, j=1,2$, of $S$. We connect this problem with the property of weak mixing for ergodic dynamical systems [24].

\section{Ergodicity: coincidence of time and ensemble averages}

We recall that if a stationary stochastic process $x(t, \lambda)$ is ergodic (see section (6), then the following equality holds for a sufficiently rich class

\footnotetext{
${ }^{3}$ The theoretical background of these experimental proposals is $p$-adic generalization of theory of probability. We also point to the recent work of Palmer [20] using $p$-adic number-theoretic approach to analysis of the Bell argument.
} 
of functions $f$

$E f(x) \equiv \int_{\Lambda} f(x(t, \lambda)) d \rho(\lambda)=E_{\text {time }} f(x) \equiv \lim _{n \rightarrow \infty} \frac{f\left(x\left(t_{1}, \lambda\right)\right)+\ldots+f\left(x\left(t_{n}, \lambda\right)\right)}{n}$.

Here $\rho$ is a probability measure (a process is ergodic with respect to some probability measure). The symbol $\Lambda$ denotes the space of chance parameters. In physical modeling related to the Bell inequality, these are hidden parameters; in classical probabilistic axiomatics, see appendix, these are so called elementary events. In particular, the law of large numbers holds true:

$$
E x \equiv \int_{\Lambda} x(t, \lambda) d \rho(\lambda)=E_{\text {time }} x \equiv \lim _{n \rightarrow \infty} \frac{x\left(t_{1}, \lambda\right)+\ldots+x\left(t_{n}, \lambda\right)}{n} .
$$

In fact, ergodicity theory was developed to generalize of the law of large numbers.

Thus, for an ergodic process, the ensemble average $E x=\int_{\Lambda} x(t, \lambda) d \rho(\lambda)$ (it does not depend on $t$, since the process is stationary) can be approximated with an arbitrary precision by the time-average with respect to almost any realization $\lambda$, see sections 6 , 7 for the mathematical formulations.

We emphasize that in all quantum experiments there are calculated the time averages. To calculate the ensemble average, one has; for example, to be able to prepare for the fixed instance of time an ensemble of quantum systems and to perform the simultaneous measurement on all these systems in a way that one measurement would not disturb another. Of course, such an experiment cannot be performed in reality. Therefore one can use the same experimental equipment and a source which generates systems one by one, but at least experimenters have to destroy possible effects of the previous trails, cf. Summhammer [17, see also [18, 19]. However, to perform such "loophole free" experiments is a complicated problem. Each experimental arrangement contains many components and in principle each of them can generate temporal correlations in data leading to violation of ergodicity. (In particular, Summhammer [17] destroyed possible memory effects only in one component of the neutron interference experiment. However, sequential dependence leading to difference between the time and ensemble averages can be generated by other components of the experimental arrangement, even by the source of neutrons.) As was pointed out, it is impossible to consider an ensemble of experimental arrangements and to prepare a single system in each of them and then 
to perform the measurement on it. It seems to be reasonable to use temporal relaxation to destroy memory effects in all components of the experimental arrangement: to prepare just one quantum system at each interval of time $\Delta t$ and to perform the measurement on it. This interval should be sufficiently large to guarantee disappearance of the memory effects.

\section{Quantum (non)ergodicity?}

In quantum mechanics we consider the chance parameter $\lambda$ labeling runs of experiments for measuring some quantum observable $x$ for systems prepared in the state $D$. Thus for any run $\lambda$ we obtain a discrete process $x\left(t_{1}, \lambda\right), \ldots, x\left(t_{n}, \lambda\right), \ldots$ (results of measurements of $x$ ). We remark that $x \equiv x^{D}(t)$, so it depends on the state $D$. In quantum formalism it is assumed that $x$ and $D$ are represented by self-adjoint operators, moreover, $D$ is positively defined and it has the unit trace (a density operator representing a quantum state). The theoretical quantum average is given by the von Neumann trace formula: $\langle x\rangle_{D}=$ Tr Dx, which is the straightforward consequence of the Born rule determining quantum probabilities.

In a huge number of experiments there was demonstrated (long before Bell's proposal) that the quantum average coincides with the time-average:

$$
\langle x\rangle_{D}=E_{\text {time }} x
$$

so

$$
\operatorname{Tr} D x=\lim _{n \rightarrow \infty} \frac{x\left(t_{1}, \lambda\right)+\ldots+x\left(t_{n}, \lambda\right)}{n} .
$$

However, besides Summhammer's experiment [17], there were no experimental results confirming quantum ergodicity, namely, coincidence of the time average $E_{\text {time }} x$ (and hence the quantum average $\langle x\rangle_{D}$ ) and the ensemble average $E x$.

\section{$4 \quad$ Nonlocality and nonergodicty?}

There were no doubts that the equality (3) should hold true even for observables considered in the EPR-Bohm experiment. One of the implicit inventions of J. Bell was assuming ergodicity of quantum mechanics and, hence, the coincidence of ensemble and time averages, 
see (11). Under such implicit assumption he could identify theoretical quantum averages (coinciding with time averages) 4 with ensemble averages with respect to the distribution of hidden variables.

The logic of such identification can be presented as follows:

- quantum averages are well approximated by time averages;

- the latter coincide (through ergodicity) with ensemble averages;

- therefore quantum averages are well approximated by ensemble averages.

In this framework Bell derived his inequality (which is an inequality for ensemble averages) and came to the conclusion that the quantum formalism is incompatible with local realism.

The crucial point of this derivation is the possibility to use the measure-theoretic representation of quantum correlations:

$$
\left\langle x_{\theta} y_{\phi}\right\rangle=\int_{\Lambda} x_{\theta}(t, \lambda) y_{\phi}(t, \lambda) d \rho(\lambda) .
$$

Here $\theta$ and $\phi$ are orientations of polarization beam splitters in labs 1,2 (separated by some distance) and $x_{\theta}, y_{\phi}$ are the corresponding quantum observables, the polarization projections. (We remind that the process is assumed to be stationary.)

The intermediate step is based on the implicit assumption of ergodicity:

$$
\int_{\Lambda} x_{\theta}(t, \lambda) y_{\phi}(t \lambda) \cdot d \rho(\lambda)=\lim _{n \rightarrow \infty} \frac{1}{n} \sum_{i=1}^{n} x_{\theta}\left(t_{i}, \lambda\right) y_{\phi}\left(t_{i}, \lambda\right),
$$

However, it has never been explicitly emphasized, neither by Bell not by thousands of his followers.

We now remark that if the stochastic process induced by measurements of polarizations of pairs of entangled photons is nonergodic, then there is no reason to identify the time and ensemble averages and hence no reason to identify the ensemble and quantum averages.

Our emphasis on "pairs" has an important mathematical background in theory of dynamical systems and especially those violating the condition of weak mixing, see section 7 . The latter condition is directly related to quantum correlation experiments of the Bell type. It guarantees ergodicity not only of the component processes, but also of

\footnotetext{
${ }^{4}$ The latter assumption is based on just the experimental experience.
} 
their pair which is used to calculate the correlation between the components. As we know from ergodicity theory (section 7), the condition of weak mixing can be violated for natural physical systems. In such a case the violation of ergodicity can be found only in experiments with compound systems.

Nowadays the hypothesis on quantum ergodicity has been confirmed just in the single experiment [17]. In this situation the use of the ergodicity assumption in derivation of the Bell inequality and its generalizations is really ad hoc.

We remark that there were presented some proofs of the Bell-type inequality which were not based on identification of the time and ensemble averages [26]-[29]. However, these proofs are heavily based on counterfactual reasoning (although the authors did not stress the latter as the crucial part of their reasoning).

Consider two experiments on measurement of photon polarizations for two pairs of angles $(\theta, \phi)$ and $\left(\theta, \phi^{\prime}\right)$. They generate two pairs of two dimensional stochastic processes, $\left(x_{\theta}(t), y_{\phi}(t)\right)$ and $\left(x_{\theta}(t), y_{\phi^{\prime}}(t)\right)$. Consider experiments with $N$ trials, for the first setting, at the moments $t_{1}, \ldots, t_{N}$ and, for the second one, at the moments $s_{1}, \ldots, s_{N}$. We get the following statistical samples

$$
\left(x_{\theta}\left(t_{1}\right), y_{\phi}\left(t_{1}\right), \ldots .,\left(x_{\theta}\left(t_{N}\right), y_{\phi}\left(t_{N}\right)\right)\right.
$$

and

$$
\left(x_{\theta}\left(s_{1}\right), y_{\phi^{\prime}}\left(s_{1}\right)\right), \ldots,\left(x_{\theta}\left(s_{N}\right), y_{\phi^{\prime}}\left(s_{N}\right)\right) .
$$

Then those who "prove" the Bell inequality without appealing to the hypothesis of ergodicity identify the statistical samples

$$
x_{\theta}\left(t_{1}\right), \ldots, x_{\theta}\left(t_{N}\right)
$$

and

$$
x_{\theta}\left(s_{1}\right), \ldots, x_{\theta}\left(s_{N}\right) .
$$

Here the counterfactual reasoning is involved. However, it is really impossible to combine peacefully such reasoning with the views of founders of quantum theory, N. Bohr and W. Heisenberg. Consideration of "experimental data" without relation to the concrete experimental context is really against the basic principle of the quantum theory - the principle of complementarity.

Such an approach was criticized by many authors, see, e.g., [30][37]. In particular, in [30] there were shown that for real stochastic 
processes, i.e., without aforementioned identification, operation with solely time averages, without identification of them with the ensemble averages, does not lead to the Bell inequality, but to its modification, i.e., the classical ensemble bound 2 is perturbed by the additional term.

\section{Can nonergodicity save realism?}

The above discussion about the fundamental role of the implicit assumption on quantum ergodicity in derivation of the Bell inequality is important for better understanding of the problem of (in)compatibility of realism and quantum theory. In a nonergodic model violation of Bell's inequality does not contradict to existence of the probability distribution of hidden variables. This was the position of De Broglie 422 in his critical analysis of the Bell argument against the local realism [15]. In fact, this position is typical for those accepting the ontic-epistemic methodology in science [39]. There are two levels of description:

- ontic,

- epistemic.

The latter description represents our knowledge about natural phenomena; the former description is about these phenomena as they are. Of course, at both levels we operate with mathematical models. Epistemic models describe observations. In particular, the quantum model is epistemic. Ontic models describe features of natural phenomena which are not approachable through observations. It seems that some philosophers treat the ontic description as representing "objective reality" as it is. However, this viewpoint does not match with the mathematical modeling approach to science. Therefore it seems to be more natural to speak not about the ontic and epistemic levels of description, but theoretical and epistemic (observational) models. This viewpoint was advertised by Hertz, Boltzmann, and later by Schrödinger, see, e.g., [40], [15]. Theoretical model need not be rigidly coupled to the observational. In this note we speculate that the quantum model describes in fact nonergodic behavior and the basic structure of the theoretical ("ontic") description, the probability distribution of hidden variables, need not be recovered from the time averages given by the quantum formalism. 


\section{Mathematical considerations: from separate ergodicity to compound non- ergodicity}

Now we recall the notion of ergodicity for stochastic processes [40]. We start with the definition of a wide-sense stationary stochastic process. Such a stochastic process, denoted by $x(t)$, has constant expectation value: $\mu_{x}=E[x(t)]$, and its autocovariance function $C_{x}(\tau)=$ $E\left[\left(x(t)-\mu_{x}\right)\left(x(t+\tau)-\mu_{x}\right)\right]$, depends only on the shift $\tau$ and not on the time variable $t$. We stress that the expectation and autocovariance function are understood as ensemble averages, not time averages, i.e., $\mu_{x}=\int x(t, \lambda) d \rho(\lambda)$ and $C_{x}(\tau)=\int\left[\left(x(t, \lambda)-\mu_{x}\right)\left(x(t+\tau, \lambda)-\mu_{x}\right)\right] d \rho(\lambda)$.

The stationary stochastic process $x(t)$ is said to be mean-ergodic (or mean-square ergodic) if the time average

$$
\langle x\rangle_{\text {time }}(T ; \lambda)=\frac{1}{T} \int_{0}^{T} x(t, \lambda) d t
$$

converges in squared mean to the ensemble average $\mu_{X}$ as $T \rightarrow \infty$. The latter means that

$$
\lim _{T \rightarrow \infty} \int\left|\frac{1}{T} \int_{0}^{T} x(t, \lambda) d t-\mu_{x}\right|^{2} d \rho(\lambda)=0 .
$$

The $L_{2}$-convergence is not precisely the type of convergence which we need to work with the experimental statistical data,. Here we need convergence almost everywhere, i.e., for almost all $\lambda$,

$$
\lim _{T \rightarrow \infty} \frac{1}{T} \int_{0}^{T} x(t, \lambda) d t=\mu_{x}
$$

This is a more advanced area of the mathematical studies about ergodicity.

Further we consider discrete stochastic processes (with sums, instead of integrals). Here (7) has the form:

$$
\lim _{n \rightarrow \infty} \frac{1}{n} \sum_{i=1}^{n} x\left(t_{i}, \lambda\right)=\mu_{x},
$$

for almost all $\lambda$. The most known and widely used result of such type was obtained by Kolmogorov for independent equally distributed random variables $x\left(t_{i}, \lambda\right)$. This is the Kolmogorov strong law of large numbers. Therefore the simplest test of ergodicity of statistical data obtained in quantum experiments is checking the independence of trials. 
If they are independent, the time and ensemble averages coincide and, hence, the quantum mechanical averages coincide with the ensemble averages, see [41] detailed analysis of this problem. However, the assumption of independent trials is too strong.

The general ergodic theorem is known as Birkhoff-Khinchin theorem. For its formulation, we need a stronger notion of stationarity based on consideration of joint probability distributions and the corresponding notion of ergodicity. A stochastic process $x(t), t \in \mathbf{N}=$ $\{1, \ldots, n, \ldots\}$, is called stationary if

$$
P((x(1), \ldots, x(n)) \in A)=P((x(1+m), \ldots, x(n+m)) \in A),
$$

for any $m$ and $n$ and any Borel subset $A$ of $\mathbf{R}^{n}$. If a process is stationary, then it is, of course, stationary in the wide-sense, but not vice versa.

Consider the space of all infinite sequences of real nu mbers

$$
\Omega=\mathbf{R}^{\mathbf{N}}=\left\{x=\left(x_{1}, \ldots, x_{k}, \ldots\right)\right\}
$$

endowed with the $\sigma$-algebra $\mathcal{F}$ generated by cylindric subsets, i.e., subsets of the form $A_{i_{1} i_{2} \ldots i_{n}}=\left\{x \in \mathbf{R}^{\mathbf{N}}:\left(x_{i_{1}} x_{i_{1}} \ldots x_{i_{n}}\right) \in A\right\}$, where $A$ is a Borel subset of $\mathbf{R}^{n}$. Consider the probability measure $P$ on this $\sigma$-algebra determined by finite dimensional probability distributions of the process $x(t)$.

In the space $\Omega$ define the left-shift operator

$$
T\left(x_{1} \ldots x_{n} \ldots\right)=T\left(x_{2} \ldots x_{n} \ldots\right) .
$$

Then the process is stationary if and only if $P\left(T^{-1}(\mathcal{A})\right)=P(\mathcal{A})$ for any $\mathcal{A} \in \mathcal{F}$.

An event $\mathcal{A} \in \mathcal{F}$ is shift-invariant if $T^{-1}(\mathcal{A})=\mathcal{A}$. The process $x(t)$ is called ergodic if every shift-invariant event $\mathcal{A}$ is trivial, i.e., $P(\mathcal{A})=0$ or 1 .

Theorem 1. (Birkhoff-Khinchin) Let the stochastic process $x(t), t=$ $1,2, \ldots$, be stationary and ergodic. If $E|x(1)|<\infty$, then (8) holds almost everywhere.

Now we consider an interesting problem of theory of ergodicity which has close relation to the identification of the ensemble averages with the time (and hence quantum-theoretical) averages in the Bell framework. Consider two stationary ergodic processes $x(t)$ and $y(t)$. Is the process $z(t)=(x(t), y(t))$ ergodic? The answer is 'no'. We remark that this implies that the product $x(t) y(t)$ of two stationary 
ergodic processes need not be ergodic. Thus in principle in quantum theory nonergodicity can be generated by measurements on compound systems. At the same time measurements of each system separately generate ergodic stationary processes. In such a case measurements on compound systems really have the special feature, nonergodicity. We can speculate that nonergodicity may be really a delicate issue. It can happen that, for each of stochastic processes $x_{\theta}(t)$ and $y_{\phi}(t)$ generated by measurements of the polarization observables in labs 1 and 2, the ensemble, time, and quantum mechanical averages coincide. But at the same time, for correlations, time and ensemble averages can be different, since the product $x_{\theta}(t) y_{\phi}(t)$ of these processes need not be ergodic. However, to derive the Bell inequality one has to operate with correlations and hence (ensemble) averages of products of stochastic processes.

\section{Mathematical considerations: cou- pling to theory of dynamical systems, weak mixing}

Turn to the representation of a stochastic process $x(t)$ by the probability distribution $P$ on the space of trajectories of this process, $\Omega=\mathbf{R}^{\mathbf{N}}$ endowed with the $\sigma$-algebra $\mathcal{F}$ generated by cylindric subsets. The iterations of the left shift map $T$ can be treated as a discrete dynamical system. (We recall that we consider only discrete time processes, $t=1,2, \ldots$.) Thus, instead of the processes, we can work with the dynamical system $(\Omega, \mathcal{F}, P, T)$. Stationarity of the stochastic process is equivalent to measure preserving property of $T$.

In turn, we can start with an arbitrary dynamical system [24] $(X, \mathcal{G}, \mu, T)$, where $\mu$ is an arbitrary probability measure on the $\sigma$ algebra $\mathcal{G}$ and $T: X \rightarrow X$ is a measurable map. We shall consider measure preserving dynamical systems. It is ergodic if every $T$-invariant event $\mathcal{A} \in \mathcal{G}$ is trivial, i.e., $\mu(\mathcal{A})=0$ or 1 .

Consider now a measurable function $f: X \rightarrow \mathbf{R}$. Such functions represent observables. Set $x_{f}(t ; \lambda)=f\left(T^{t} \lambda\right), \lambda \in X$. Then $x_{f}(t)$ is a stochastic process.

Thus we can speak either about stochastic processes or dynamical systems. For latter, we formulate the famous Birkhoff theorem:

Theorem 2. Let $(X, \mathcal{G}, \mu, T)$ be an ergodic dynamical system. 
Then, for function $f$, such that $E|f|<\infty$,

$$
\lim _{n \rightarrow \infty} \frac{1}{n} \sum_{i=1}^{n} f\left(T^{i} \lambda\right)=\int_{X} f(\lambda) d \rho(\lambda)
$$

almost everywhere.

In particular, if we consider the dynamical system $(\Omega, \mathcal{F}, P, T)$ corresponding to the stochastic process $x(t)$, then we can select $f\left(x_{1}, \ldots, x_{n}, \ldots\right)=$ $x_{1}$. For this map, we have $x_{f}(t ; \lambda)=x(t ; \lambda)$.

However, for many purposes, the ergodicity constraint is too weak and typically there are considered dynamical systems satisfying stronger constraint known as mixing. A dynamical system $(X, \mathcal{G}, \mu, T)$ is called strongly mixing if, for any $A, B \in \mathcal{G}$,

$$
\lim _{n \rightarrow \infty} \mu\left(A \cap T^{-n} B\right)=\mu(A) \mu(B),
$$

where $T^{-n} B \in \mathcal{G}, T$ is measurable. 5 For our aim, it is enough to consider a weaker property. A dynamical system is said to be weak mixing if

$$
\lim _{n \rightarrow \infty} \frac{1}{n} \sum_{k=0}^{n-1}\left|\mu\left(A \cap T^{-k} B\right)-\mu(A) \mu(B)\right|=0 .
$$

Strong mixing implies weak mixing which in turn implies ergodicity. However, inverse is not valid.

Theorem 3. A dynamical system $(X, \mathcal{G}, \mu, T)$ is weakly mixing if and only if, for any ergodic dynamical system $(Y, \mathcal{G}, \nu, Q)$, the direct product of these dynamical systems is also ergodic.

Thus in our analysis of the Bell argument a violation of the Bell inequality can (but need not) be coupled to violation of weak mixing condition.

${ }^{5}$ Now by ignoring the problem of measurability consider, instead of $T$-preimages, direct $T$-images. Then by taking into account that $\mu(X)=1$, we have:

$$
\lim _{n \rightarrow \infty} \frac{\mu\left(T^{n} A \cap B\right)}{\mu(B)}=\lim _{n \rightarrow \infty} \mu\left(T^{n} A \mid B\right)=\mu(A)=\frac{\mu(A)}{\mu(X)} .
$$

In a shaker there are two liquids, wine and water, initially wine was concentrated in the volume $A$. So, what is the proportion of wine in an arbitrary volume $B$ of the shaker? It is the same as the original proportion of wine in the shaker, so it is doing really good mixing. 
The evident objection to this kind of reasoning is that, for the Bell states, it is natural to assume that stochastic processes observed in lab 1 and lab 2 are based on the same dynamical system $(X, \mathcal{G}, \mu, T)$ and one can play only with selection of functions $f_{1}$ and $f_{2}$ determining observables. Of course, matching of the latter assumption to the real experimental situation is not eveident at all. But we shall not go in such a discussion about matching. In fact, mathematics gives us the formal argument implying that even identification of two dynamics (determining the temporal structure of stochastic processes observed in lab 1 and lab 2) does not change the previous consideration about coupling of weak mixing with a violation of the Bell inequality.

Theorem 4. A dynamical system $(X, \mathcal{G}, \mu, T)$ is weakly mixing if and only if its the direct product with itself is also ergodic.

Thus even if the dynamics of each of entangled photons in the Bell experiment is described by the same dynamical map $T^{t}$ which is ergodic by itself, then the dynamics of the pair of these photons need not be ergodic (if this dynamical map is not weak mixing). I agree that there is a kind of mystery in disappearance of ergodicity for a compound system. However, this is not physical, but mathematical mystery. It was discussed a lot in mathematical literature devoted to theory of stochastic processes and dynamical systems. There is no possibility to go deeper into the corresponding mathematical discussion in this paper. So, further analysis of consequences of coupling of the Bell argument and spurious quantum nonlocality with theory of mixing and ergodicity will be presented in a future paper. In particular, if the hypothesis on violation of ergodicity for processes generated by measurements on compound quantum systems is correct, then we have to understand coupling between entanglement and violation of weak mixing. This is the exciting problem.

Finally, we remark that coupling of the theory of dynamical systems with interference phenomena and violation of Bell's inequality was considered in papers of a few authors, see, e.g., [42], [43].

\section{Concluding remarks}

A violation of the Bell-type inequalities predicted by the quantum formalism and confirmed by a series of experiments need not be interpreted as incompatibility of local realism and quantum theory; they can be as well interpreted as evidences of a violation of ergodicity 
for quantum measurement process. Under the assumption of nonergodicity one can proceed without rejecting realism. However, the latter is closely coupled to structuring physics through two levels of description of physical phenomena, ontic-epistemic, or two mathematical models, theoretical-observational. The hypothesis on quantum nonlocality (spooky action at the distance) can be rejected either as an absurd assumption (see Einstein, Podolsky, and Rosen [10]) or by using Occam's Razor.

We remark that GHZ-like tests, which in some aspects present a stronger claim for nonlocality than Bell's arguments, can also be analyzed from the viewpoint of violation of the hypothesis of ergodicity. Such analysis is based on the contextual representation of these tests [44]. However, this is a separate and complicated problem which deserves special consideration.

We finally make the following remark about possible future experiments in quantum foundations. It would be natural to switch the experimental research in quantum foundations from the study of probabilistic behavior of outputs of measurements for entangled system to experiments that can test "pure" (not mixed with nonlocality) ergodicity. The next step is testing the hypothesis on weak mixing, but not through consideration of compound systems and the the pairs of processes generated by measurement on the sub-systems. In fact, weak mixing can be checked straightforwardly for noncompound sys-

tems. (However, for the moment I do not have the real experimental proposals.)

This paper was partially supported by the EU-project Quantum Information Access and Retrieval Theory (QUARTZ), Grant No. 721321.

\section{References}

[1] Bell, J.: Speakable and Unspeakable in Quantum Mechanics. Cambridge Univ. Press, Cambridge (1987).

[2] M. Genovese, Research on hidden variable theories: A review of recent progresses, Physics Reports 413, 319-396 (2005).

[3] B. Hensen et al, Experimental loophole-free violation of a Bell inequality using entangled electron spins separated by $1.3 \mathrm{~km}, \mathrm{Na}-$ ture 526, 682 (2015). 
[4] M. Giustina et al, A significant-loophole-free test of Bell's theorem with entangled photons, Phys. Rev. Lett. 115, 250401 (2015).

[5] L. K. Shalm et al, A strong loophole-free test of local realism, Phys. Rev. Lett. 115, 250402 (2015).

[6] G. Adenier and A. Yu. Khrennikov, J. Phys. B 40131141 (2007).

[7] A. Bednorz, Analysis of assumptions of recent tests of local realism. Phys. Rev. A 95, 042118 (2017).

[8] G. Adenier and A. Khrennikov, Test of the no-signaling principle in the Hensen loophole-free CHSH experiment. Fortschritte der Physik (Progress in Physics). doi: 10.1002/prop.201600096 .

[9] M. Kupczynski, Is Einsteinian No-signalling violated in Bell Tests? https://arxiv.org/ftp/arxiv/papers/1709/1709.00708.pdf

[10] Einstein, A., Podolsky, B. Rosen, N.: Can quantum-mechanical description of physical reality be considered complete?. Phys. Rev. 47 (10), 777-780 (1935).

[11] Bohr, N.: Can quantum-mechanical description of physical reality be considered complete? Phys. Rev. 48, 696-702 (1935)

[12] A. Aspect, Closing the Door on Einstein and Bohr's Quantum Debate, Physics 8, 123 (2015)

[13] H. Wiseman, Quantum physics: Death by experiment for local realism. Nature 526, 649650 (2015).

[14] Kupczynski, Can Einstein with Bohr Debate on Quantum Mechanics Be Closed? arXiv:1603.00266 [quant-ph].

[15] A. Khrennikov, After Bell. Fortschritte der Physik (Progress in Physics). doi: 10.1002/prop.201600044.

[16] V. Buonomano, Quantum uncertainties, Recent and Future Experiments and Interpretations, edited by W.M. Honig, D.W. Kraft and E. Panarella, NATO ASI Series, 162, Plenum Press, New York (1986).

[17] J. Summhammer, Neutron interferometric test of the nonergodic interpretation of quantum mechanics. Il Nuovo Cimento, 103 B, 265-280 (1989).

[18] A. Yu. Khrennikov, p-adic probability predictions of correlations between particles in the two slit and neuron interferometry experiments. Il Nuovo Cimento B, 113, 751-760 (1998). 
[19] A. Yu. Khrennikov, Description of experiments detecting $p$-adic statistics in quantum diffraction experiments. Doklady Mathematics (Dokl. Russian Academy of Sc.), 58, N. 3, 478-480 (1998).

[20] T. Palmer, $p$-adic distance, finite precision and emergent superdeterminism: A number-theoretic consistent-histories approach to local quantum realism. arXiv:1609.08148 [quant-ph]

[21] A. Khrennikov, Frequency analysis of the EPR-Bell argumentation. Found. Phys. 32, 1159-1174 (2002).

[22] H. De Raedt, K. Michielsen, K. Hess, Irrelevance of Bell's Theorem for experiments involving correlations in space and time: a specific loophole-free computer-example. Computer Physics Communications 209, 42 - 47 (2016).

[23] A. N. Shiryaev, Probability. Springer, Berlin, 1996.

[24] D. V. Anosov, Ergodic theory. In M. Hazewinkel (ed.), Encyclopedia of Mathematics, Springer Science and Business Media B.V., Kluwer Academic, Dordrecht, 2001.

[25] A. Yu. Khrennikov, Quantum nonlocality or nonergodicity? A critical study of Bell's arguments. arXiv:quant-ph/0512178.

[26] H.P. Stapp, Phys. Rev. D 3, 1303 (1971).

[27] P. H. Eberhard, Nuovo Cim. SSB, 75 (1977).

[28] P.H. Eberhard, Nuovo Cim. 46B, 392 (1978).

[29] Ph. H. Eberhard, Phys. Rev. A 477-750 (1993).

[30] A. Khrennikov, Interpretations of Probability. VSP Int. Sc. Publishers, Utrecht/Tokyo, 1999; De Gruyter, Berlin, 2009, second edition (completed).

[31] A. Khrennikov, Frequency derivation of the EPR-Bohm correlations. Il Nuovo Cimento, 119, N. 2, 131-147 (2004).

[32] A. Khrennikov, Ensemble fluctuations and the origin of quantum probabilistic rule. J. Math. Phys., 43, N. 2, 789-802 (2002).

[33] De Muynck, W. M.: Interpretations of quantum mechanics, and interpretations of violations of Bell's inequality. In: Khrennikov, A.Yu. (ed) Foundations of Probability and Physics, pp. 95-104. Series PQ-QP: Quantum Probability and White Noise Analysis 13. WSP, Singapore (2001).

[34] De Muynck, W. M.: Foundations of Quantum Mechanics, an Empiricists Approach. Kluwer Academic Publ., Dordrecht (2002) 
[35] Hess, K. and Philipp, W.: Exclusion of time in Mermin's proof of Bell-type inequalities. In: Khrennikov, A.Yu. (ed) Quantum Theory: Reconsideration of Foundations-2, pp. 243-254. Ser. Math. Model. 10, Växjö University Press, Växjö (2003)

[36] Hess, K. and Philipp, W.: Bell's theorem: critique of proofs with and without inequalities. In: Adenier, G., Khrennikov, A. Yu. (eds): Foundations of Probability and Physics-3, pp. 150-155. American Institute of Physics, Ser. Conference Proceedings 750, Melville, NY (2005).

[37] K. Hess, H. De Raedt and K. Michielsen, Hidden assumptions in the derivation of the theorem of Bell. In: FQMT'11: Frontiers of Quantum and Mesoscopic Thermodynamics (Prague, Czech Republic, 2530 July 2011), T. M. Nieuwenhuizen, P. D. Keefe and V. Spicka, Physica Scripta, T151, 01400 (2012).

[38] L. de Broglie and E. Silva J. Andrade (the author of a chapter) 1964 The Current Interpretation of Wave Mechanics. A Critical Study (Amstredam-London-New York: Elsevier)

[39] Atmanspacher, H. and Primas, H.: Epistemic and ontic quantumrealities. In: Adenier, G., Khrennikov, A. Yu. (eds) Foundations of Probability and Physics-3, pp. 49-62. American Institute of Physics, Ser. Conference Proceedings 750, Melville, NY (2005)

[40] S. D'Agostino, Continuity and completeness in physical theory: Schrödinger's return to the wave interpretation of quantum mechanics in the 1950's. In: Bitbol, M. and O. Darrigol (eds.) E. Schrödinger: Philosophy and the Birth of Quantum Mechanics, 339-360. Editions Frontieres, Gif-sur-Yvette (1992)

[41] M. Kupczynski, H. De Raedt, Breakdown of statistical inference from some random experiments, Computer Physics Communications, 200, 168-175 (2016).

[42] D. Dubischar, V. M. Gundlach, O. Steinkamp, A. Khrennikov, The interference phenomenon, memory effects in the equipment and random dynamical systems over the fields of p-adic numbers. Il Nuovo Cimento, B, 114, N. 4, 373- 382 (1999).

[43] T. N. Palmer, Bell's conspiracy, Schrödinger's black cat and global invariant sets. Phil. Trans. R. Soc. A 373, 20140246 (2015).

[44] A. Khrennikov, Contextualist viewpoint to Greenberger-HorneZeilinger paradox. Phys. Lett., A, 278, 307-314 (2001). 